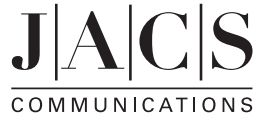

Published on Web 12/23/2003

\title{
DNA-Templated Self-Assembly of Protein and Nanoparticle Linear Arrays
}

\author{
Hanying Li, Sung Ha Park, John H. Reif, Thomas H. LaBean, ${ }^{*}$ and Hao Yan* \\ Department of Computer Science, Duke University, Durham, North Carolina 27708
}

Received September 4, 2003; E-mail: hy1@cs.duke.edu; thl@cs.duke.edu

Recent years have witnessed substantial advances in the use of DNA as a smart material to construct periodically patterned structures. ${ }^{1}$ DNA also has been designed to direct the assembly of other functional molecules by the use of appropriate attachment chemistries. ${ }^{2}$ The diversity of materials which can be chemically attached to DNA considerably enhances the attractiveness of DNA nanostructures for assembly of other materials. Self-assembling DNA tiling lattices represent a versatile system for nanoscale construction. The methodology of DNA lattice self-assembly begins with the chemical synthesis of single-stranded DNA molecules, which self-assemble into DNA branched motif complexes, known as tiles. ${ }^{1 \mathrm{~b}-1 \mathrm{f}}$ DNA tiles can carry sticky-ends that preferentially match the sticky-ends of other particular DNA tiles, thereby facilitating the further assembly into lattices. Self-assembled twodimensional DNA tiling lattices composed of tens of thousands of tiles have been demonstrated. ${ }^{1 \mathrm{~b}-1 \mathrm{f}}$ Self-assembled DNA arrays provide an excellent template for spatially positioning other molecules with increased relative precision and programmability.

Here we report an experiment using a linear array of DNA triple crossover molecules (TX) to controllably template the self-assembly of two forms (single-layer or double-layer) of streptavidin linear arrays through biotin-streptavidin interaction. Figure 1 illustrates the design. The TX molecule used here was derived from the DNA motif described elsewhere, ${ }^{1 \mathrm{c}}$ and it consists of seven oligonucleotides hybridized to form three double-stranded helices lying in a plane and linked by strand exchange at four immobile crossover points. The TX molecule shown in Figure 1a is designed such that it contains two stem loops protruding, one each out of the upper and the lower helices. A linear array of the TX molecules can be obtained by designing three pairs of sticky ends where their complementarity is represented by matching color and geometric shape (Figure 1b). To template the assembly of streptavidin molecules, the hairpin loops were modified to incorporate two biotin groups per loop, indicated by the small blue dots. Formation of single-layer or double-layer streptavidin linear arrays was controlled using two different templates which are illustrated in Figure 1b. In the first template (left panel), only one stem loop in each TX molecule was modified with biotin groups. However, in the second template (right panel), both stem loops were modified to incorporate biotins. The binding of streptavidin molecules, which is represented as yellow dots, to the two different templates resulted in singlelayer or double-layer streptavidin linear arrays, as shown in Figure $1 b$.

Streptavidin has a diameter of $\sim 4 \mathrm{~nm}$. Its binding to the selfassembled TX array generates bumps at biotinylated locations on hairpin loops of the TX tiles which can be detected by atomic force microscopy imaging (AFM). Figure 2a shows an AFM image of a sample containing only streptavidin, demonstrating that the streptavidin molecules are randomly distributed on the surface. Figure $2 b$ shows an AFM image of the bare TX linear DNA array. The length of each hairpin loop is $\sim 3.4 \mathrm{~nm}$ (10 base pairs) and is not resolved due to the well-known limitation of the lateral resolution of AFM.

418 - J. AM. CHEM. SOC. 2004, 126, 418-419

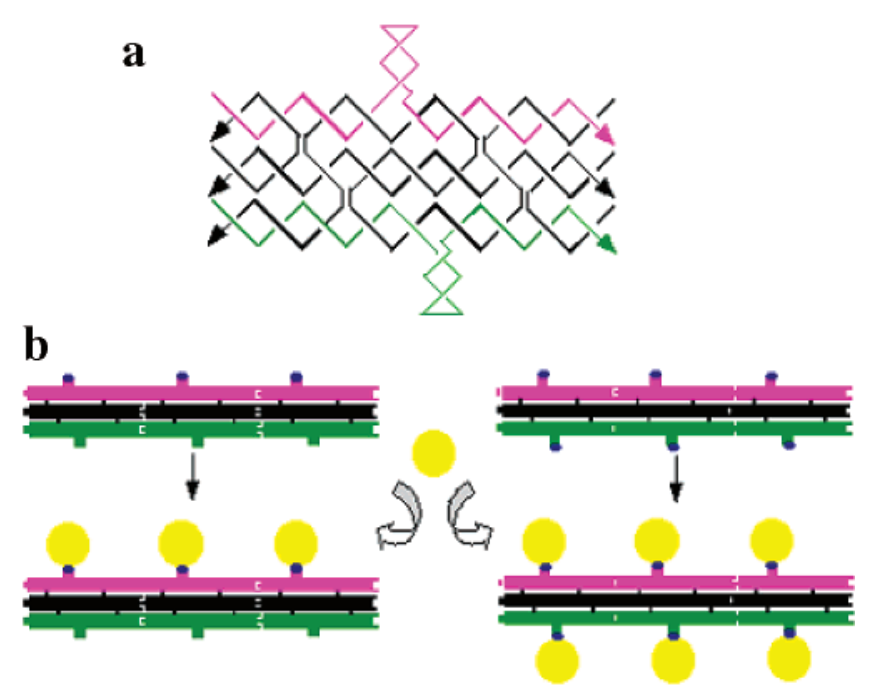

Figure 1. Schematic drawing of the TX DNA-templated self-assembly of streptavidin linear arrays.
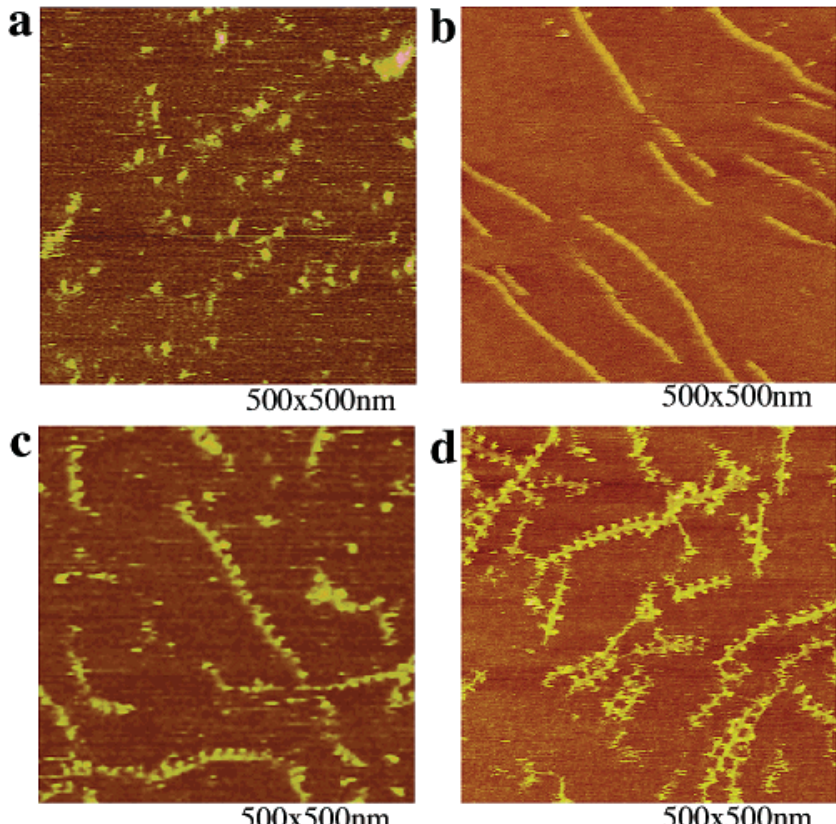

Figure 2. AFM images of the DNA-templated protein arrays. (a) streptavidin alone. (b) bare TX DNA tile arrays (c) single-layer streptavidin array (d) double-layer streptavidin array.

However, the binding of $4 \mathrm{~nm}$ streptavidin to each biotinylated hairpin loop dramatically enhances its visibility by AFM. Figure $2 \mathrm{c}$ shows an AFM image of the TX-templated single-layer streptavidin linear arrays, where only one side loop in each TX tile is modified with biotin. The streptavidin molecules appeared periodically on one side of the array. The measured distance between each adjacent streptavidin molecules is $\sim 17 \mathrm{~nm}$, matching 

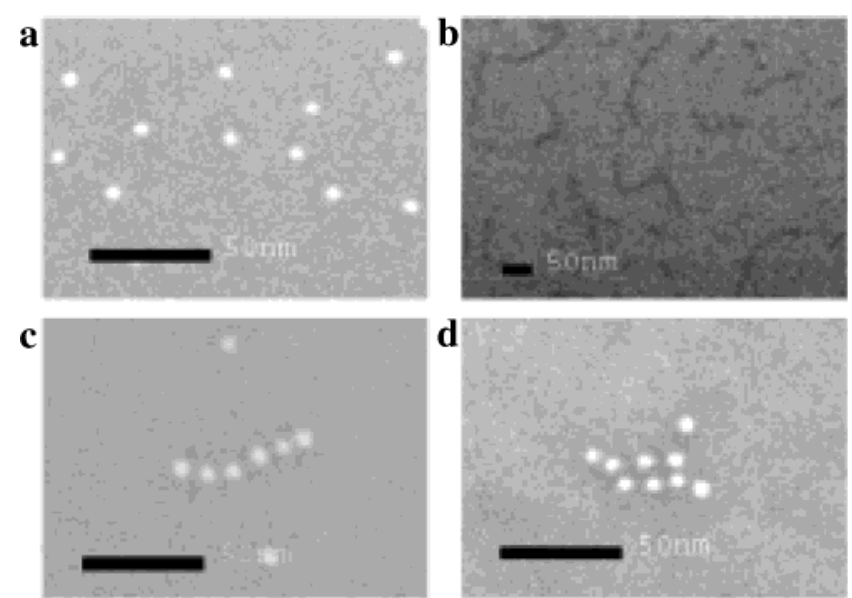

Figure 3. SEM images of the DNA-templated streptavidin-nanogold arrays, scale bars: $50 \mathrm{~nm}$. (a) streptavidin-gold alone. (b) bare TX DNA arrays (c) single-layer streptavidin-gold arrays (d) double-layer streptavidin-gold arrays.

the designed distance between each two adjacent repeating hairpin loops along the linear TX arrays. AFM height measurements (see Supporting Information) show that the streptavidin has a height of $\sim 3.8 \mathrm{~nm}$, compared to the height of $\sim 1.8 \mathrm{~nm}$ measured on bare DNA. This further confirms that the periodic bumps in the linear array resulted from the binding of streptavidin molecules. Doublelayer streptavidin linear arrays were also obtained by replacing only one strand in the first template to incorporate biotin groups on both hairpin loops. The AFM image in Figure 2d shows the formation of the double-layer protein linear array illustrated in Figure 1b, demonstrating the programmability of DNA nanostructures for templating of protein arrays. Since streptavidin is tetravalent (capable of binding four biotin molecules), the relative concentrations of DNA assembly and streptavidin protein were adjusted to ensure each array contained only a single layer of DNA lattice. At lower relative streptavidin concentrations, cross-linked superstructures containing one protein layer sandwiched between two DNA layers were observed (see Supporting Information).

One potential application of DNA nanotechnology is the use of self-assembled DNA lattices to scaffold assembly of nanoelectronic components, especially metallic nanoparticles. Here we demonstrate the use of the linear TX arrays for the assembly of streptavidinconjugated 5-nm gold particles, where the gold can be precisely positioned periodically on the self-assembled DNA array. Panels c and $d$ of Figure 3 are scanning electron microscopy (SEM) images showing the TX array templated self-assembly of single-layer and double-layer streptavidin-gold arrays. These assemblies can be compared to the SEM images of randomly distributed streptavidinconjugated gold nanoparticles (Figure 3a) and bare TX DNA assemblies (Figure 3b). The distance between adjacent gold particles in the single-layer and double-layer arrays is $\sim 17 \mathrm{~nm}$, matching the designed structures. We note that the average length of observed linear arrays incorporating gold nanoparticles is decreased compared to the average length of streptavidin arrays without gold. Possible explanations for this trend include repulsion between neighboring gold nanoparticles carrying electrical charges, decreased biotinstreptavidin binding affinity following gold conjugation, or steric constraints due to the additional mass of the attached gold. Further experiments are required to differentiate between these mechanisms.

In summary, we were able to use self-assembled DNA nanostructures to precisely control the spatial location of both streptavidin molecules and their nanogold conjugates. The specific biotinstreptavidin interaction, combined with the programmability of DNA nanostructures, may lead to more complex patterned structures with addressable features. The organization of streptavidinconjugated gold nanoparticles into periodic arrays templated by DNA nanostructures provides a convenient way to construct multiple nanoparticle arrays for electrical measurements ${ }^{3}$ by increasing the size of the nanogold. It may also find applications in constructing logical molecular electronic devices such as quantum cellular automata, ${ }^{4}$ or serve as interconnects between other nanoelectronic and molecular electronic components by providing uniformly sized gaps between adjacent gold nanoparticles.

Acknowledgment. This work has been supported by grants from NSF to H.Y., T.H.L., and J.H.R. (EIA-00-86015, EIA-0218376, EIA-0218359) and DARPA/AFSOR to J.H.R. (F30602-01-2-0561).

Supporting Information Available: Materials and methods, details of DNA sequences, AFM height measurements, AFM images of crosslinked DNA - streptavidin arrays, additional SEM images (PDF). This material is available free of charge via the Internet at http://pubs.acs.org.

\section{References}

(1) (a) Seeman, N. C. Nature 2003, 421, 427-430. (b) Winfree, E.; Liu, F. L. Wenzler, A.; Seeman, N. C. Nature 1998, 394, 539-544. (c) LaBean, T. H.; Yan, H.; Kopatsch, J.; Liu, F.; Winfree, E.; Reif, J. H.; Seeman, N. C. J. Am. Chem. Soc. 2000, 122, 1848-1860. (d) Mao, C.; Sun, W.; Seeman, N. C. J. Am. Chem. Soc. 1999, 121, 5437-5442. (e) Sha, R.; Liu, F.; Millar, D. P.; Seeman, N. C. Chem. Biol. 2000, 7, 743-749. (f) Yan, H.; et al. Science 2003, 301, 1882-1884. (g) Yan, H.; LaBean, T. H.; Feng, L.; Reif, J. H. Proc. Natl. Acad. Sci. U.S.A. 2003, 100, 81038108 .

(2) (a) Alivisatos, P. A.; et al. Nature 1996, 382, 609-611. (b) Taton, T. A.; Mirkin, C. A.; Letsinger, R. L. Science 2000, 289, 1757-1760. (c) Jin, R.; Wu, G.; Li, Z.; Mirkin, C. A.; Schatz, G. C. et al. J. Am. Chem. Soc. 2003, 125, 1643-1654. (d) Braun, E.; Eichen, Y.; Sivan, U.; Ben-Yoseph, G. Nature 1998, 391, 775-778. (e) Niemeyer, C. M.; Burger, W.; Peplies, J. Angew. Chem., Int. Ed. 1998, 37, 2265-2268. (f) Loweth, C. J.; et al. Angew. Chem., Int. Ed. 1999, 38, 1808-1812. (g) Zanchet, D.; Micheel, C. M.; Parak, W. J.; Gerion, D.; Alivisatos, A. P.; Nano Lett. 2001, 1, 32-35. (h) Patolsky, F.; Weizmann, Y.; Lioubashevski, O.; Willner, I. Angew. Chem., Int. Ed. 2002, 41, 2323-2326. (i) Monson, C. F.; Woolley, A. T. Nano Lett. 2003, 3, 359-362 (j) Xiao, S.; et al. J. Nanopart. Res. 2002, 4, 313-317.

(3) Bezryadin, A.; et al. Appl. Phys. Lett. 1999, 74, 2699-2701.

(4) (a) Orlov, A. O.; et al. Science 1997, 277, 928-930. (b) Amlani, I.; et al. Science 1999, 284, 289-291.

JA0383367 